\title{
Editorial: Radiosurgical induced malignancy associated with stereotactic radiosurgery
}

\author{
Adomas Bunevicius $^{1} \cdot$ Styllianos Pikis ${ }^{1}$ • David Schlesinger ${ }^{1}$. Jason Sheehan ${ }^{1,2}$ \\ Received: 30 November 2020 / Accepted: 1 December 2020 / Published online: 6 January 2021 \\ (C) The Author(s), under exclusive licence to Springer-Verlag GmbH, AT part of Springer Nature 2021
}

Stereotactic radiosurgery (SRS) has become an integral component of contemporary neurosurgical and radiation oncology practices worldwide allowing safe and effective management of malignant and benign central nervous system disorders. Ongoing advancements in SRS planning and delivery techniques are expected to further contribute to improving safety and effectiveness of SRS procedures [3]. Greater adoption of SRS therapies requires healthcare providers to better understand potential long-term risks and adverse events associated with SRS that would allow them to choose the most optimal treatment strategies and facilitate the most transparent patient counseling. There is an increasing interest in potential adverse radiation events associated with radiation scattering to organs outside the treated volume [6].

Paddick with colleagues compared extracranial radiation doses from SRS and also estimated the lifetime risk of radiation-induced malignancy after SRS between different treatment platforms [8]. In 20 patients undergoing SRS with Gamma Knife Perfexion, the authors measured extracranial surface radiation doses at distances of 18,43 , and $75 \mathrm{~cm}$ from the target, corresponding to the approximate positions of the thyroid, breast and gonads. Comparative data for other SRS platforms were identified via literature review and excess lifetime cancer risk from this exposure was estimated using the National Cancer Institute (NCI) RadRAT calculator. The authors identified significant variability in extracranial radiation doses between SRS platforms that translated into a wide range of lifetime risk of extracranial radiation-induced malignancy. There was also appreciable between-subject variability in extracranial radiation doses and lifetime malignancy risk in patients treated with Gamma Knife

Jason Sheehan

jsheehan@virginia.edu

1 Department of Neurological Surgery, University of Virginia, Charlottesville, VA, USA

2 Health Sciences Center, Box 800212, Charlottesville, VA 22908, USA
Perfexion unit. This preliminary study draws our attention to potential long-term risks of extracranial malignancies associated with SRS for CNS disorders

Data describing the clinically realized lifetime risk of extracranial secondary malignancies from intracranial SRS remains relatively sparse, and the uncertainties remain large. Cohort studies in adults [1] and children [7] have found the incidence of secondary cancers in long-term survivors is approximately $9 \%$. However, only a small proportion $(\sim 8 \%)$ of these can be directly attributed to radiotherapy. The relatively low and uncertain risks of secondary malignancy must be balanced with acute risk posed by the immediate neurological disease and the short-term management strategy.

The carcinogenic potential of ionizing radiation is not limited to whole-body exposures. The risk of radiosurgery-induced intracranial malignancies or malignant transformation of the treated lesion remains a major subject of investigation and debate. However, radiosurgery associated malignancies seem to be rare occurrences. A multicenter study of 4905 patients who underwent SRS for benign intracranial pathologies reported a cumulative incidence of radiation-associated malignancy of $0.00045 \%$ (95\% CI $0.00-0.34$ ) over 10 years, which was similar to the incidences of primary malignant brain tumors in the general population [9]. In a retrospective study of 1142 patients following SRS for benign intracranial pathologies, no cases of radiation-induced tumors were noted. Malignant transformation occurred in 7 meningioma patients and in one patient with vestibular schwannoma. The post-radiosurgery malignant transformation risk was $0.5 \%$ (95\% CI, $0.0-0.9 \%$ ), $0.8 \%$ (95\% CI, 0.0 $1.8 \%$ ), and $2.4 \%(95 \% \mathrm{CI}, 0.0-5.5 \%)$ at 5,10 , and 15 years respectively [10]. The apparently low risk of SRS induced intracranial neoplasia must be weight against the risks of resection and the natural history of untreated intracranial disease.

More work remains to reduce the uncertainty in estimates of extracranial secondary malignancy risk to a level that would make a reference level workable. Resources such as the report of AAPM Task Group 158 [6] provide guidelines for measuring and reporting peripheral radiation doses across 
various modalities and can serve as a reference for future studies. The comparative data presented in the study highlights the importance of considering SRS technique when trying to minimize extracranial radiation dose. When using linear accelerator-based SRS, radiation delivery using vertex fields may increase dose along the long axis of the body. Highly modulated static-field intensity-modulated radiation therapy (IMRT) techniques tend to have higher monitor units (MUs) and thus higher whole-body radiation dose due to leakage radiation [6]. These extracranial dose factors may be reduced by avoiding vertex fields and employing updated techniques such as multiple lesion, single isocenter volumetric modulated arc therapy (VMAT) where the total MU/treatment time does not increase with the number of targets [4]. However the tradeoff may be increasing mid-range doses to normal brain [5]. Proton and charged particle radiosurgery may result in lower integral dose to the brain [2]. However, more research is required to better understand the magnitude of extracranial dose due to neutron production in these modalities.

In summary, Paddick and colleagues have presented an important step forward in our understanding of the extracranial doses delivered with contemporary intracranial SRS platforms. As radiosurgery utilization expands and outcomes after SRS continue to improve for patients afflicted with benign and malignant conditions, further work is needed to quantify and reduce the dose delivered outside the target volume and thereby mitigate risks to normal intracranial and extracranial structures.

\section{References}

1. Berrington de Gonzalez A, Curtis RE, Kry SF, Gilbert E, Lamart S, Berg CD, Stovall M, Ron E (2011) Proportion of second cancers attributable to radiotherapy treatment in adults: a cohort study in the US SEER cancer registries. Lancet Oncol 12(4):353-360
2. Boehling NS, Grosshans DR, Bluett JB, Palmer MT, Song X, Amos RA, Sahoo N, Meyer JJ, Mahajan A, Woo SY (2012) Dosimetric Comparison of Three-Dimensional Conformal Proton Radiotherapy, Intensity-Modulated Proton Therapy, and IntensityModulated Radiotherapy for Treatment of Pediatric Craniopharyngiomas. Int J Radiat Oncol Biol Phys 82(2):643-652

3. Bunevicius A, Sheehan D, Lee Vance M, Schlesinger D, Sheehan JP (2020) Outcomes of Cushing's disease following Gamma Knife radiosurgery: effect of a center's growing experience and era of treatment. J Neurosurg:1-8

4. Clark GM, Popple RA, Young PE, Fiveash JB (2010) Feasibility of single-isocenter volumetric modulated arc radiosurgery for treatment of multiple brain metastases. Int J Radiat Oncol Biol Phys 76(1):296-302

5. Hossain S, Keeling V, Hildebrand K, Ahmad S, Larson DA, Sahgal A, Ma L (2016) Normal Brain Sparing With Increasing Number of Beams and Isocenters in Volumetric-Modulated Arc Beam Radiosurgery of Multiple Brain Metastases. Technol Cancer Res Treat 15(6):766-771

6. Kry SF, Bednarz B, Howell RM, Dauer L, Followill D, Klein E, Paganetti H, Wang B, Wuu C-S, Xu XG (2017) AAPM TG 158: Measurement and calculation of doses outside the treated volume from external-beam radiation therapy. Medical Physics 44(10):e391-e429

7. Meadows AT, Friedman DL, Neglia JP, Mertens AC, Donaldson SS, Stovall M, Hammond S, Yasui Y, Inskip PD (2009) Second neoplasms in survivors of childhood cancer: findings from the Childhood Cancer Survivor Study cohort. J Clin Oncol 27(14): 2356-2362

8. Paddick I, Cameron A, Dimitriadis A (2020) Extracranial dose and the risk of radiation-induced malignancy after intracranial stereotactic radiosurgery. Is it time to establish a therapeutic reference level? J Neurosurg:1-8. https://doi.org/10.3171/2019.12. JNS192743

9. Sheehan J, Yen CP, Steiner L (2006) Gamma knife surgeryinduced meningioma. Report of two cases and review of the literature. J Neurosurg 105(2):325-329

10. Wolf A, Naylor K, Tam M et al (2019) Risk of radiation-associated intracranial malignancy after stereotactic radiosurgery: a retrospective, multicentre, cohort study. Lancet Oncol 20(1):159-164

Publisher's note Springer Nature remains neutral with regard to jurisdictional claims in published maps and institutional affiliations. 